\title{
Índices para análise de redes de transporte urbano de passageiros de alta capacidade ${ }^{1}$
}

\section{Indexes for rapid transit network analysis}

\author{
Isoda, Marcos Kiyoto de Tani e'; Mori, Klara Kaiser ${ }^{2}$
}

\begin{abstract}
1 Faculdade de Arquitetura e Urbanismo da Universidade de São Paulo; Programa de Pós-Graduação em Planejamento Urbano e Regional; São Paulo, Brasil; marcos.isoda@usp.br.

2 Faculdade de Arquitetura e Urbanismo da Universidade de São Paulo; Professora Livre-Docente de Planejamento Urbano e Regional;

kmkaiser@usp.br.
\end{abstract}

\begin{abstract}
RESUMO
O objetivo é apresentar o potencial do uso de indicadores como ferramentas de projeto e avaliação de redes de transporte de passageiros de alta capacidade através de um conjunto de aplicações práticas. O objeto de estudo é a rede ferroviária da Região Metropolitana de São Paulo (RMSP), administradas pela Companhia Paulista de Trens Metropolitanos (CPTM) e pela Companhia do Metropolitano de São Paulo (Metrô). Este artigo apresenta a aplicação destes indicadores à rede existente e aos principais planos recentes. Conclui-se que o uso destes indicadores é desejável em etapas preliminares e de planejamento estratégico de macroescala.
\end{abstract}

Palavras-chave: Planejamento Urbano. Planejamento de Transporte. Metrô. Trem Metropolitano. Rede de Alta Capacidade.

\begin{abstract}
The objective is to present the potential of indexes as tools for the design and evaluation of rapid transit networks through a set of applications. The object of study is the rail network of the Metropolitan Region of São Paulo (RMSP), administered by Companhia Paulista de Trens Metropolitanos (CPTM) and Companhia do Metropolitano de São Paulo (Metrô). This paper presents these indexe's application to existing network and recente plans. It concludes that the use of the indexes is desirable in the preliminary stages and of strategic macro-scale planning.
\end{abstract}

Keywords: Urban Planning. Transit Planning. Rapid Transit. Metropolitan Railway. Rapid Transit Network.

\footnotetext{
${ }^{1}$ ISODA, Marcos Kiyoto de Tani e; MORI, Klara Kaiser. Índices para análise de redes de transporte urbano de passageiros de alta capacidade. In: II SIMPÓSIO NACIONAL DE GESTÃO E ENGENHARIA URBANA: SINGEURB, 2019, São Paulo. Anais... Porto Alegre: ANTAC, 2019
} 


\section{INTRODUÇÃO}

O objetivo deste artigo é apresentar o potencial do uso de indicadores como ferramentas de avaliação de redes de transporte de passageiros de alta capacidade através de um conjunto de aplicações práticas. O objeto de estudo é a rede ferroviária da Região Metropolitana de São Paulo (RMSP), administradas pela Companhia Paulista de Trens Metropolitanos (CPTM) e pela Companhia do Metropolitano (Metrô).

Primeiramente serão apresentados os conjuntos de indicadores e sua descrição. Na sequência, será apresentada a sua aplicação na rede metroferroviária atual da RMSP e os principais projetos recentes, seguida das conclusões.

\section{INDICADORES PARA AVALIAÇÃO DE REDES}

Este estudo apresenta o potencial do uso de indicadores para a avaliação espacial das redes de alta capacidade, partindo do conceito de alta capacidade - uma rede segregada e de linhas exclusivas voltada para o transporte de grandes demandas de passageiros. A ideia é obter parâmetros quantitativos de avaliação da qualidade de uma rede, que podem ser utilizados tanto para análise de sistemas existentes quanto como parâmetro para novas propostas. Os índices aqui expostos visam complementar a etapa de avaliação das redes em sua etapa inicial de forma mais simples e ágil comparado à modelagem matemática, ferramenta mais utilizada, porém de alto custo de operação.

Serão apresentados três conjuntos de indicadores, visando a quantificação de características espaciais das redes de alta capacidade. Apesar de simples, quando aplicados em conjunto permitem uma leitura bastante rica.

Os primeiros indicadores são a extensão total da rede e a sua proporção em relação à população da aglomeração urbana ( $\mathrm{km} /$ habitante).

O segundo conjunto busca caracterizar a forma da rede através da correlação entre o comprimento médio de linhas e o número de estações ${ }^{2}$. As redes são classificadas em três formas principais: cobertura local, acessibilidade regional, e cobertura regional.

O terceiro conjunto busca quantificar o nível de estruturação interna da rede, em dois subgrupos. Primeiro, os indicadores oriundos da Teoria dos Grafos ${ }^{3}$. Em seguida, o índice conexões por linha, com resultados análogos aos índices dos grafos ${ }^{4}$.

\subsection{Extensão da rede}

Estes dois indicadores, a extensão total da rede e a sua proporção em relação à população da aglomeração urbana, representam a ordem de grandeza do sistema e a relação com a população. Abstraem os aspectos geométricos e a forma de distribuição da rede. Resultam em informações de fácil leitura, motivo pelo qual são muito usados (SORT, 2005), mas também frequentemente criticados.

\subsection{Forma da rede}

Aplicaremos aqui um par de índices espaciais para análise de redes de metrô proposto por Derrible \& Kennedy (2010), que buscam caracterizar a forma e traçar diferentes modelos de rede. Entre as desvantagens estão a abstração da geometria da rede e a necessidade de quantificação de todas as estações (o que muitas vezes não ocorre em planos).

Do cruzamento deste conjunto de indicadores são sugeridas três formas de rede: "cobertura local", de grande densidade central; "acessibilidade regional", linhas longas e menor número de estações; e "cobertura regional", de grande extensão e grande densidade de estações

\footnotetext{
2 DERRIBLE \& KENNEDY, 2010.

${ }^{3}$ GATTUSO \& MIRIELLO, 2005; DERRIBLE \& KENNEDY, 2010.

${ }^{4}$ ISODA, 2013.
} 
(vide gráfico) ${ }^{5}$. Às redes analisadas por DERRIBLE \& KENNEDY acrescentamos outras redes (quadrados vazados). Podemos extrair daqui que, do ponto de vista da distribuição espacial: (1) há mais de um modelo de rede possível e (2) eles não distinguem os dois modos aqui estudados. De fato, as redes de trens testadas - CPTM, RER e S-Bahn - possuem características espaciais muito distintas.

Figura 1 - Formas de Rede

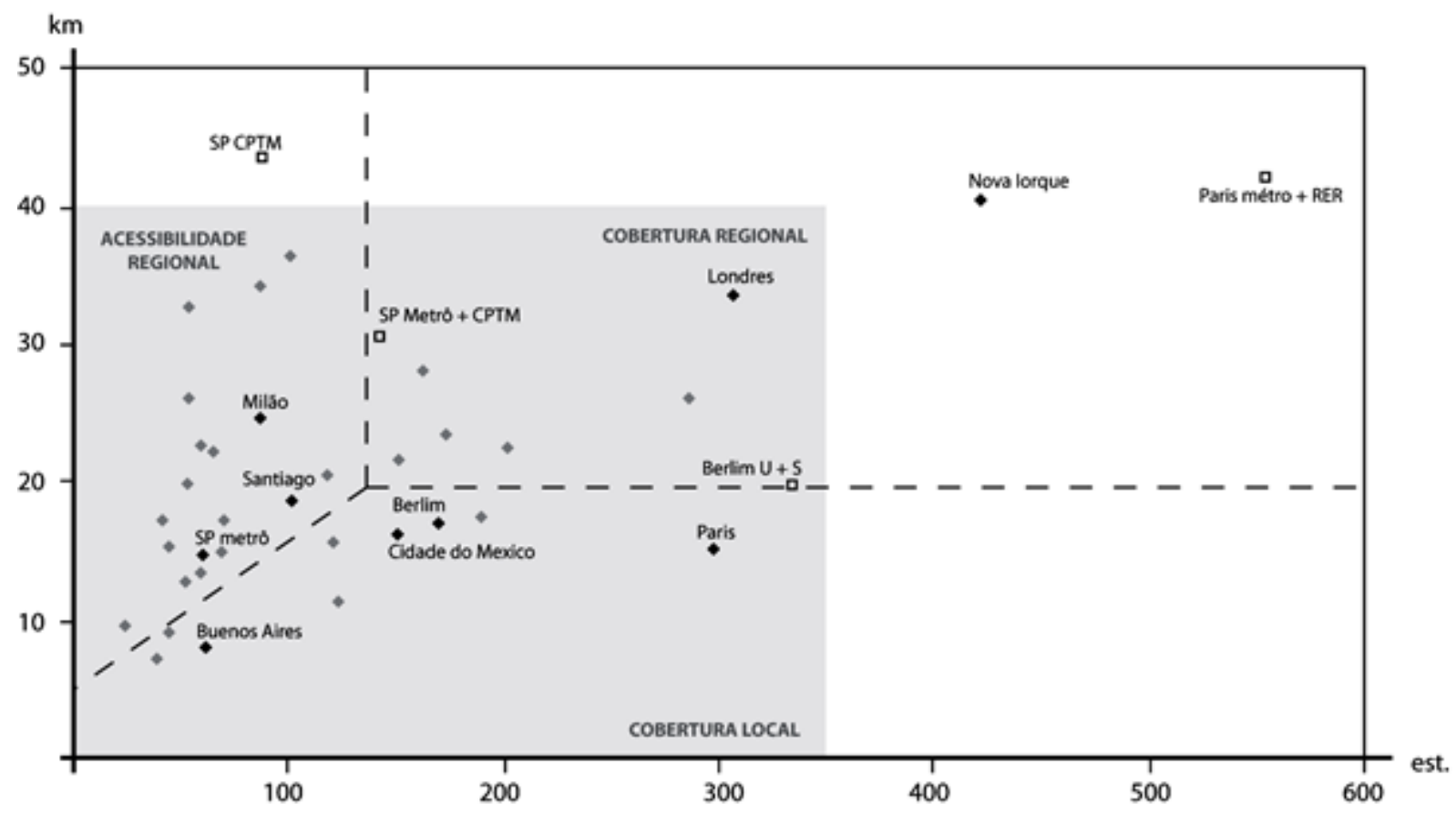

Fonte: Os autores, sobre Derrible \& Kennedy, 2010

\subsection{Estruturação da rede - índice de complexidade}

O último conjunto de indicadores buscam quantificar a forma de estruturação geométrica da rede, ou seja, o quanto as diferentes linhas se conectam entre si. Para isso são utilizados os grafos. A conversão de redes de alta capacidade em um grafo para a análise macroestrutural é um procedimento bastante simples, basta converter as estações em vértices e os trechos entre estações em arestas.

Seguimos a recomendação de Derrible \& Kennedy (2010) de considerar como vértices apenas as estações terminais (peso 1) e de transferência (peso maior que 2) ${ }^{6}$. A partir dele calculamos os índices de complexidade, conectividade e o número de loops ${ }^{7}$. Dos três indicadores, adotamos o de complexidade como o mais representativo8.

\subsection{Estruturação da rede - conexões por linha}

Buscando uma análise simples e preliminar da estruturação de uma rede foi criado o índice de conexões por linha (C/L), que pode ser calculado sobre qualquer mapa simples de rede.

${ }^{5} \mathrm{O}$ gráfico original abrangia uma área menor (aqui hachurada) e excluía a rede de Nova lorque (apesar de quantificada no estudo citado), evidenciando que ela é um caso excepcional (possui muitos compartilhamentos de via, o que contraria o princípio de linhas independentes, tornando-a um caso complicado de analisar). Mesmo casos considerados paradigmáticos como de Londres e Paris são casos extremos de seus grupos.

${ }^{6}$ Para mais detalhes, conferir DERRIBLE \& KENNEDY (2010), e também GATTUSO \& MIRIELLO (2005). Estes últimos fazem o mesmo tipo de análise utilizando todas as estações das redes (vértices de peso 2), obtendo resultados numericamente diferentes, mas relativamente similares.

${ }^{7}$ As fórmulas de cálculo destes índices são triviais na Teoria dos Grafos e sua dedução está disponível nos livros do assunto.

${ }^{8}$ Os três indicadores possuem forte correlação. Ver Derrible \& Kennedy (2010) e Gattuso \& Miriello (2005). 
A principal dificuldade é em casos de redes com linhas sem separação operacional clara, onde há ramais ou compartilhamentos de via (sistemas que abrem mão do conceito de linhas exclusivas).

O objetivo deste índice é uma análise similar aos índices dos grafos. Esta correlação foi verificada pelo cruzamento do índice de conexões por linha com o índice de complexidade, cuja regressão linear resultou satisfatória $\left(R^{2}=0,9083\right)$.

\section{ANÁLISES DA REDE DE ALTA CAPACIDADE DA RMSP}

Apresentaremos uma leitura dos planos recentes para a rede de alta capacidade da RMSP utilizando o conjunto de indicadores apresentado. As redes de metrô propostas possuem diferentes modelos, variando de uma rede central e densa a uma aberta e abrangente, 0 que transparece nos índices?.

Figura 2 - Rede de alta capacidade atual

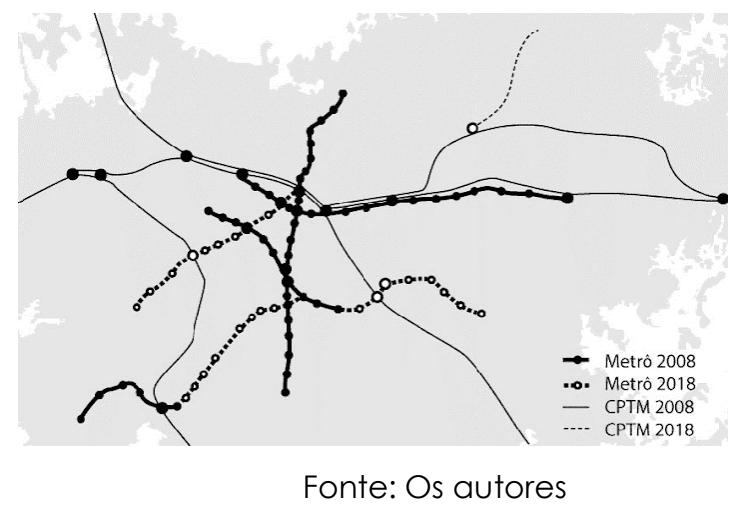

Além da rede atual, serão analisadas as principais redes propostas e avaliadas pelos planos recentes: Plano Integrado de Transportes Urbanos para 2020 (Pitu 2020); Rede Essencial /Plano Integrado de Transportes Urbanos para 2025 (Pitu 2025); e Atualização da rede metropolitana de alta e média capacidade de transporte da RMSP (Rede 2030).

Figura 1 - Proposta do PITU 2020 (Rede Aberta)

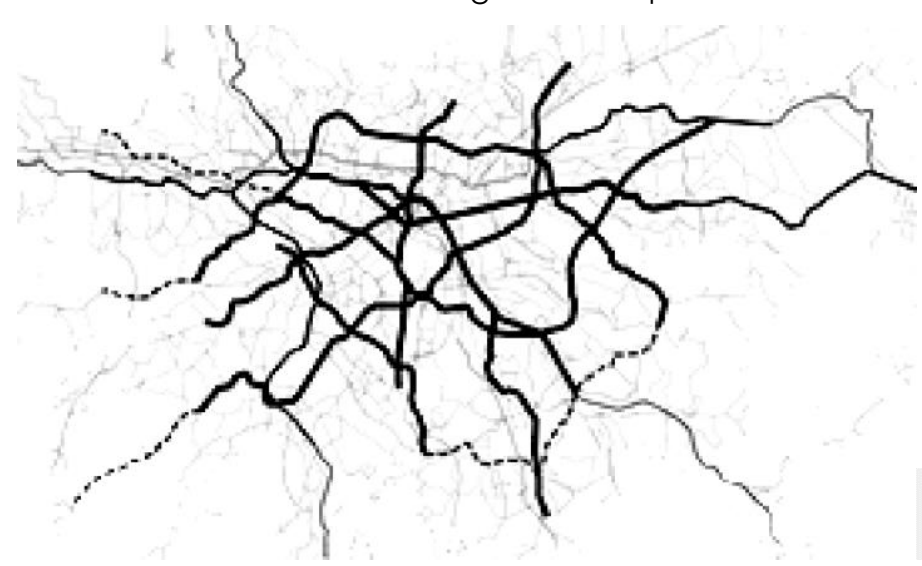

PITU 2020 - REDE ABERTA TREM APROXIMADOR MEERÓ MEVEO $_{\text {LEVE }}$

Fonte: Os autores, sobre STM, 1999

\footnotetext{
${ }^{9}$ As propostas para a rede da CPTM não variam muito em sua extensão física e nem sempre são precisas em seus limites, o que impossibilita o cálculo dos índices. Por esse motivo foram calculados os indicadores para as redes de metrô apenas.
} 
Figura 1 - Rede do PITU 2025 (Rede Essencial)

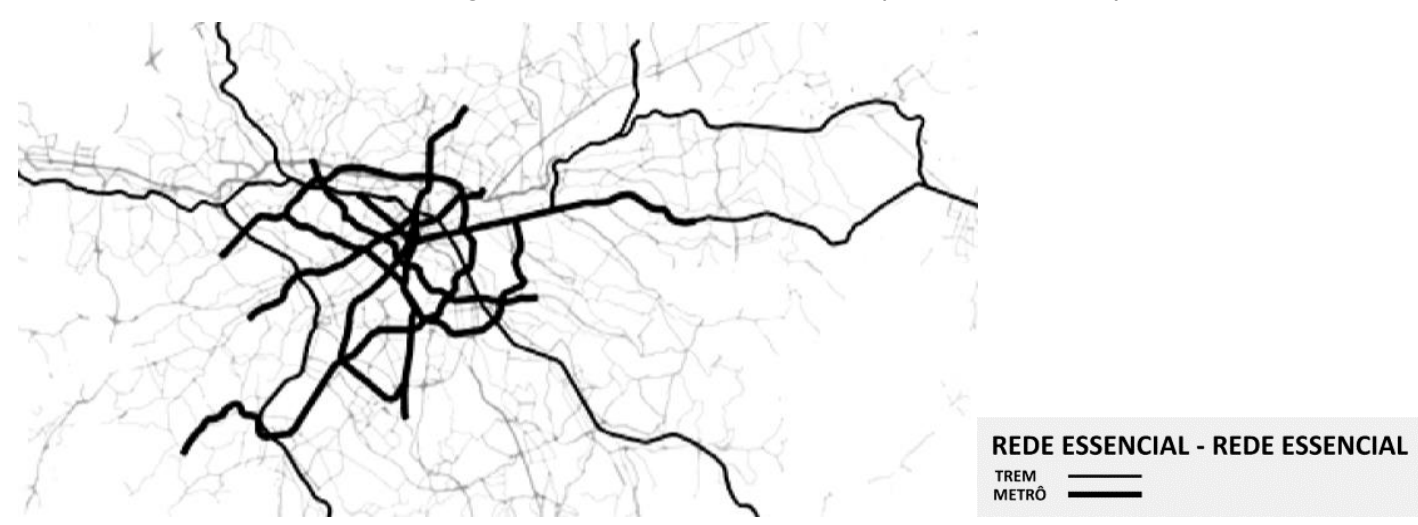

Fonte: Os autores, sobre STM, 2006

Figura 1 - Rede de alta capacidade para 2030

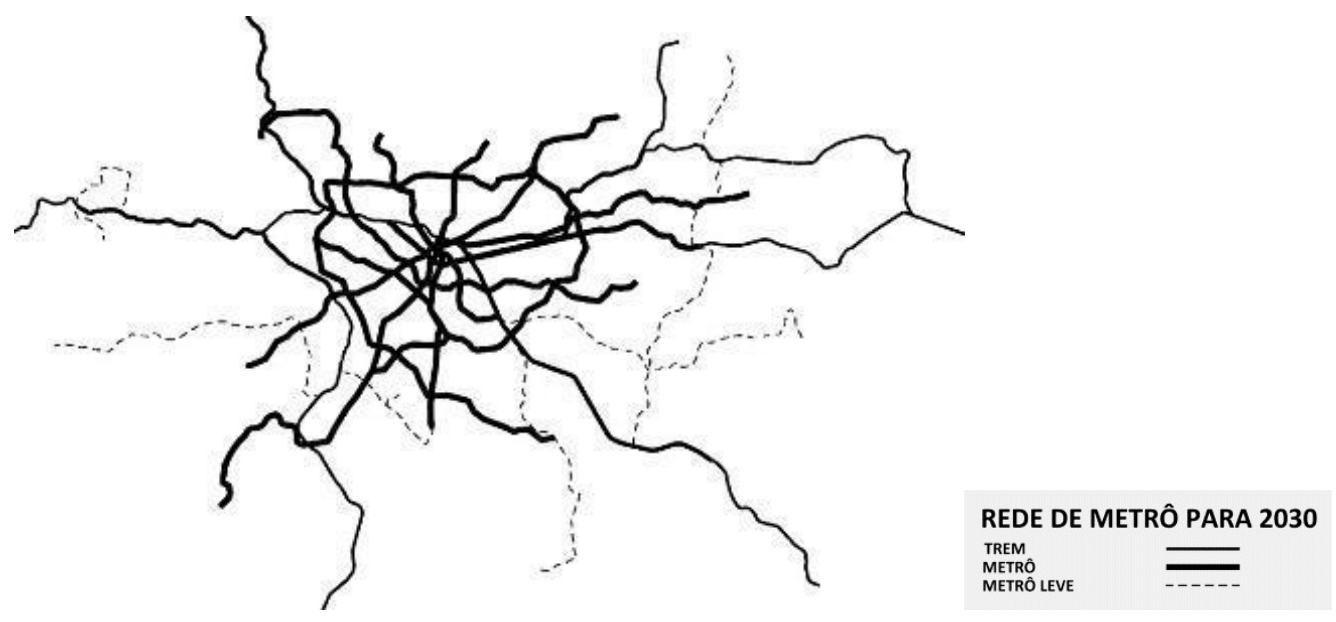

Fonte: Os autores, sobre STM, 2013

Tabela 1 - Índices dos planos recentes para o Metrô de São Paulo

\begin{tabular}{ccccccc}
\hline Rede & $\begin{array}{c}\text { Ano } \\
\text { Horizonte }\end{array}$ & $\begin{array}{c}\text { Linha } \\
\text { média }\end{array}$ & Estações & $\begin{array}{c}\text { Km/ } \\
\text { mi hab }\end{array}$ & Complexidade & c/L \\
\hline Atual - Metrô & 2018 & 14,8 & 63 & 3,7 & 1,07 & 1,2 \\
\hline Atual - Metrô + CPTM & 2018 & 30,4 & 147 & 16,7 & 1,41 & 1,5 \\
\hline Pitu 2020 Rede Aberta & 2020 & 26,3 & 145 & 10,6 & 1,41 & 2,6 \\
\hline Pitu 2025 - Rede Essencial & 2025 & 20,4 & 128 & 7,3 & 1,50 & 3,0 \\
\hline Rede 2030 & 2030 & 22,9 & 236 & 11,0 & 1,53 & 2,9 \\
\hline
\end{tabular}

Fonte: Os autores

Pelos índices de complexidade e de conexões por linha (c/L) percebemos que os planos procuram criar uma rede mais estruturadas do que a atual (índices de Complexidade acima de 1,4 e c/L acima de 2,5), similares a algumas das grandes metrópoles mundiais (ISODA, 2013).

O índice de quilometragem de rede por milhão de habitantes dos planos está em torno de 10, nenhuma se aproxima dos índices das grandes metrópoles mundiais (Londres, Paris, Berlim, Nova lorque), em torno de $20-30 \mathrm{~km} / \mathrm{mi}$ hab (ISODA, 2013). É importante lembrar que este é o único dos índices utilizados que leva em consideração aspectos a aglomeração urbana. 
Estes indicadores apontam para o nível de estruturação interna da rede, mas não permitem uma definição de modelos de rede. Para essa análise utilizamos a categorização de formas de rede, a partir do número de estações e do comprimento médio de linhas.

Figura 1 - Formas de rede dos planos recentes para o Metrô de São Paulo



Fonte: Os autores, sobre Derrible \& Kennedy, 2010

Pelo gráfico percebemos que as três propostas apresentam modelos distintos, porém numa gama de variação condizente dentro do universo previamente apresentado.

O comprimento médio de linhas se mantêm por volta dos $20 \mathrm{Km}$, o que permite discutir objetivamente modelos de rede, como vemos nos casos mundiais: $15 \mathrm{~km}$ para Paris; $33 \mathrm{~km}$ para Londres; $40 \mathrm{~km}$ para Nova lorque; $16 \mathrm{~km}$ para Cidade do México; e $9 \mathrm{~km}$ para Buenos Aires.

\section{CONCLUSÕES}

Dado o alto custo de implantação dos sistemas de transporte de alta capacidade, existe a necessidade de utilizar métodos que busquem quantificar o seu desempenho. Os índices apresentados procuram dar uma leitura simples e direta para fatores específicos numa etapa preliminar.

Conclui-se que o uso destes indicadores é desejável em etapas preliminares e de planejamento estratégico de macroescala. Acreditamos no potencial analítico e propositivo destes índices, cujo uso foi esboçado neste estudo. Através destes índices é possível estabelecer padrões de serviço desejáveis, como uma quilometragem de rede por habitante ou um padrão de estruturação espacial da rede.

A proporção de rede por habitante representa de alguma maneira o quanto a rede pode atender da população, abstraindo completamente os aspectos espaciais; a complexidade e a conectividade das redes permite avaliar a qualidade da articulação espacial da rede, porém abstrai completamente a aglomeração urbana. Cabe destaque para o uso de índices para quantificar aspectos espaciais das redes, aspecto pouco explorado ainda, seja pela literatura científica, seja no campo prático.

Este conjunto de indicadores permite perceber que: (1) há variação nos modelos de rede propostos; (2) ainda que apresentem redes mais bem estruturadas do que a atual, em geral não propõe um patamar desejável para uma das maiores metrópoles mundiais; e (3) caso 
alguma das propostas houvesse sido implantada em sua totalidade, teríamos uma rede mais eficiente do que a existente.

Por trás de tudo isso está a busca por analisar racionalmente fenômenos que são sociais, econômicos e culturais, que possuem componentes que nem sempre são exatos.

\begin{abstract}
"Vale destacar a redução dos diferenciais de preço do solo da aglomeração urbana, isto é, redução dos preços relativos das localizações mais privilegiadas (...). Trata-se aqui, na verdade, do instrumento mais efetivo - se não o único de combate à especulação imobiliária descontrolada, frequentemente enunciado e visto como um dos grandes objetivos do planejamento urbano" (DEÁK, 1999, p. 337)
\end{abstract}

A distribuição espacial dos investimentos em infraestruturas é uma das formas principais de transformação da estrutura urbana presente (DEÁK, 1999). A distribuição dos investimentos em infraestrutura urbana buscando o aumento da acessibilidade seria então a principal forma de redução das desigualdades e da segregação socioespacial.

\title{
AGRADECIMENTOS
}

Agradecemos à Fundação de Amparo à Pesquisa do Estado de São Paulo (FAPESP) pelo financiamento da pesquisa de mestrado que deu origem a este artigo.

\section{REFERÊNCIAS}

COMPANHIA DO METROPOLITANO DE SÃO PAULO. Evolução da Rede Básica do Metrô: 19681985. São Paulo: Metrô, 1986.

COMPANHIA DO METROPOLITANO DE SÃO PAULO. Rede Essencial: Trechos Prioritários. São Paulo: Metrô. 2006.

DEÁK, Csaba. "Elementos de uma política de transportes para São Paulo", in: DEÁK, Csaba \& SCHIFFER, Sueli (org.). O Processo de Urbanização no Brasil. São Paulo: Edusp, 1999.

DERRIBLE, Sybil \& KENNEDY, Christopher. "Characterizing metro networks: state, form, and structure", in: Transportation 37. 2010.

GATTUSO, Domenico \& MIRIELLO, Ernesto. "Compared Analysis of Metro Networks Supported by Graph Theory", in: Networks and Spatial Economics, 5. Springer, 2005.

ISODA, Marcos Kiyoto de Tani e. Transporte sobre trilhos na Região Metropolitana de São Paulo: estudo sobre a concepção e a inserção das redes de transporte de alta capacidade. São Paulo: Dissertação de Mestrado FAUUSP. 2013.

SÃO PAULO (Estado), Secretaria de Estado dos Transportes Metropolitanos. Pitu 2020: Plano Integrado de Transportes Urbanos para 2020. São Paulo: STM, 1999.

SÃO PAULO (Estado), Secretaria de Estado dos Transportes Metropolitanos. Pitu 2025: Plano Integrado de Transportes Urbanos para 2025. São Paulo: STM, 2006.

SÃO PAULO (Estado), Secretaria de Estado dos Transportes Metropolitanos. Atualização da rede metropolitana de alta e média capacidade de transporte da RMSP. São Paulo: STM, 2013.

SORT, Jordi Juliá. Redes Metropolitanas (Metropolitan Networks). Barcelona: Gustavo Gili. 2005.

VUCHIC, Vukan. Urban Transit: Systems and Technology. Hoboken: John Wiley \& Sons, 2007.

VUCHIC, Vukan. Urban Transit: Urban transit: Operations, Planning and Economics. Hoboken: John Wiley \& Sons, 2005. 